\title{
Impacto de la Caries en la Calidad de Vida Relacionada con la Salud Oral de Adolescentes: Revisión Sistemática y Metaanálisis
}

\author{
Impact of Caries on Oral Health Related Quality of Life \\ in Adolescents: A Systematic Review and Meta-Analysis
}

\author{
Andrea Matamala-Santander'; Fernando Rivera-Mendoza² \& Carlos Zaror ${ }^{3,4}$
}

\begin{abstract}
MATAMALA-SANTANDER, A.; RIVERA-MENDOZA, F. \& ZAROR, C. Impacto de la caries en la calidad de vida relacionada con la salud oral de adolescentes: Revisión sistemática y metaanálisis. Int. J. Odontostomat., 13(2):219-229, 2019.

RESUMEN: Las caries constituye un importante problema de salud pública, dada su alta prevalencia y consecuencias en la calidad de vida de los adolescentes. El objetivo de esta revisión sistemática y metaanálisis es evaluar el impacto de las caries dental en la calidad de vida relacionada con salud oral (CVRSO) en adolescentes. Se efectuó una búsqueda sistemática en las bases de datos de MEDLINE, EMBASE, Cochrane, ScieLo y Lilacs. Se incluyeron estudios primarios publicados en inglés, español y portugués, que evalúen CVRSO en adolescentes con caries usando instrumentos validados. Dos investigadores de manera independiente realizaron el proceso de selección y extracción de datos. La herramienta Effective Public Health Practice Project fue usada para evaluar el riesgo de sesgo de los estudios incorporados. Se usaron modelos de efectos aleatorios para estimar el efecto combinado para datos continuos y categóricos. La búsqueda combinada identificó 1.152 artículos, de los cuales 29 estudios (34 artículos) cumplían con los criterios de inclusión. Veintiocho estudios tenían un diseño de corte transversal y solo uno era de cohorte. El riesgo de sesgo fue clasificado en la mayoría de los casos como débil (18/29). Los pacientes con caries presentaban significativamente más probabilidades de reportar un compromiso de la CVRSO que los controles (OR=2,50, 95 \% IC: 1,47-4,26). Los dominios más afectados fueron el funcional (Diferencia de medias $(M D)=0,74,95 \%$ IC: 0,27-1,20) y el psicológico $(M D=0,73,95$ \% IC:0,21-1,26). Esta revisión proporciona evidencia de que las caries tienen un impacto negativo en la CVRSO de los adolescentes.
\end{abstract}

PALABRAS CLAVE: caries dental, calidad de vida, adolescentes.

\section{INTRODUCCIÓN}

La caries es una de las enfermedades crónicas más prevalentes a nivel mundial y la cuarta más costosa en cuanto a tratamiento (Glick et al., 2012). Entre sus consecuencias más frecuentes podemos mencionar el dolor intenso asociado comúnmente a ausentismo escolar en adolescentes (Krisdapong et al., 2013) la alteración del desarrollo normal de funciones tan cotidianas como la masticación y el compromiso de la estética producto de la pérdida de dientes. En consecuencia, las lesiones dentinarias no tratadas se encuentran asociadas a una menor calidad de vida relacionada con salud oral en adolescentes (Severo Alves et al., 2013).
Estudios epidemiológicos sobre salud oral y factores de riesgo constituyen una herramienta básica para la planificación de programas de prevención, control e intervención (Garcia et al., 2015). Sin embargo la mayoría de estos estudios sólo utilizan medidas clínicas. La calidad de vida relacionada con salud oral (CVRSO) es cada vez más considerada en la investigación de los servicios de salud para evaluar tendencias y necesidades en salud oral basadas en los requerimientos de la población (Sischo \& Broder, 2011). La CVRSO es un constructo multidimensional que representa la magnitud en que la vida diaria de un individuo se ve afectada por problemas orales (Baker,

\footnotetext{
${ }^{1}$ Estudiante Magíster en Odontología, Facultad de Odontología, Universidad de La Frontera, Temuco, Chile.

${ }^{2}$ Centro de Investigación en Odontología Legal y Forense (ClO), Facultad de Odontología, Universidad de La Frontera, Temuco, Chile.

${ }^{3}$ Departamento de Odontopediatría y Ortodoncia. Facultad de Odontología. Universidad de la Frontera, Chile.

${ }^{4}$ Centro de Investigación en Epidemiología, Economía y Salud Públcia Oral (CIEESPO), Facultad de Odontología, Universidad de La Frontera, Temuco, Chile.
} 
2007). La incorporación de la medición de la CVRSO en la evaluación integral de la salud oral es de suma importancia porque proporciona información esencial, al considerar las necesidades de tratamiento de individuos y poblaciones desde su propia perspectiva. También es una importante herramienta para la evaluación de intervenciones, servicios y programas de salud pública (Jokovic et al., 2002; Palencia et al., 2014) en especial aquellos que incluyen niños y adolescentes, dado que constituyen uno de los principales grupos objetivo de los servicios dentales (Montero et al., 2018).

La adolescencia es un período que abarca desde los 10 hasta los 19 años, de acuerdo a lo establecido por la OMS, en el cual no sólo se produce un crecimiento y maduración física acelerada sino que también se verifican cambios psicológicos, sexuales, de comportamiento y socialización. La adolescencia es un proceso continuo y complejo de naturaleza biopsicosocial a través del cual los adolescentes construyen, un conjunto de capacidades y aptitudes que les permiten satisfacer sus necesidades para alcanzar un estado de completo bienestar. Por lo anteriormente mencionado, es indispensable que su calidad de vida sea buena, que pueda cubrir sus necesidades, para que el adulto del mañana viva y se desarrolle individual y socialmente (Casas López, 2010).

Varios estudios de corte transversales han demostrado el impacto negativo de la caries en la CVRSO entre niños y adolescentes de diferentes poblaciones en todo el mundo (Tubert-Jeannin et al., 2005; Foster Page et al., 2005, 2008; Turton et al., 2015; RamosJorge et al., 2015; Martins et al., 2015; Corrêa-Faria et al., 2016), sin embargo, hasta donde tenemos conocimiento, no existe una revisión sistemática que evalúe y cuantifique el impacto de la caries en la CVRSO de adolescentes. Por lo tanto, el objetivo de este estudio fue evaluar el impacto de la caries en la CVRSO de adolescentes y determinar los dominios que se ven más afectados al sintetizar la evidencia disponible a través de un enfoque de revisión sistemática.

\section{MATERIAL Y MÉTODO}

Protocolo y Registro. Se efectuó una revisión sistemática de la literatura, de acuerdo a los lineamientos de PRISMA (Preferred Reporting Items for Systematic Reviews and Meta-Analyses) (Moher et al., 2009). Este estudio se encuentra registrado en PROSPERO bajo el siguiente código CRD42016038927.
Criterios de selección. Se incluyeron estudios en inglés, español y portugués que evaluaran e informaran la CVRSO de adolescentes, usando instrumentos validados para medir la CVRSO al igual que para el diagnóstico de caries y presentaran los resultados para pacientes con y sin caries por separado.

Se excluyeron estudios realizados en pacientes medicamente comprometidos, que utilizaran instrumentos genéricos para evaluar calidad de vida relacionada con salud oral; incluyera pacientes de otras edades (diferentes a la de la población objetivo) y no entregara los resultados estratificados por edad; reportes de casos y series de casos con una muestra menor a 10 individuos; estudios secundarios y finalmente estudios no realizados en humanos.

Fuentes de información y búsqueda. Se realizó una búsqueda sistemática en octubre de 2017, usando Medline (desde 1966 a octubre de 2017), Embase (desde 1974 a octubre de 2017), Biblioteca Cochrane (La Biblioteca Cochrane 2017), Lilacs (desde 1982 a octubre de 2017) y SciELO (desde 1998 a octubre 2017). La estrategia de búsqueda utilizada en PubMed fue: ((((("Dental Caries"[Mesh]) OR "DMF Index"[Mesh]) OR (((((tooth) OR teeth) OR dental)) AND (((((caries) OR

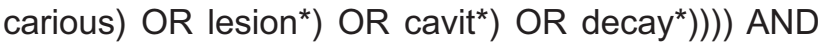
((((("Quality of Life"[Mesh]) OR Quality of Life[tiab]) OR HRQoL[tiab]) OR OHRQoL[tiab]) OR QoL[tiab])) AND (((()(("Child"[Mesh]) OR "Infant"[Mesh]) OR "Adolescent"[Mesh]) OR infant*) OR child*) OR teen*) OR adolescent $\left.{ }^{*}\right)$. Todas las referencias identificadas se extrajeron en una base de datos de EndNote X6 para facilitar su administración y eliminar duplicados.

Proceso de selección de los estudios. Dos miembros del equipo de estudio (AMS y FRM) revisaron de forma independiente los artículos encontrados en las búsquedas bibliográficas, examinándolos en tres fases consecutivas: títulos, resúmenes y revisión de textos completos utilizando el software en línea COVIDENCE (www.covidence.org). Un tercer revisor (CZ) resolvió las discrepancias.

Proceso de recolección de datos. La extracción de datos y la evaluación del riesgo de sesgo de los estudios fue realizada por dos revisores (AMS y FRM) utilizando un formulario de recolección de datos predeterminado y estandarizado. Se realizó una prueba piloto en 6 artículos potencialmente elegibles para homogeneizar los criterios entre los revisores a lo largo de todo el proceso. Los revisores no se encontraban 
enmascarados a los autores. Finalmente, se verificó la lista de referencias de los artículos seleccionados para identificar otros posibles estudios que pudieran incluirse. La codificación de los criterios de inclusión y exclusión se definió y registró en cada fase.

La información extraída fue: datos de publicación, diseño del estudio, tamaño de la muestra, características del paciente, criterios de diagnóstico de caries, instrumento utilizado para evaluar CVRSO y los resultados obtenidos de cada grupo evaluado (frecuencia del evento, media y desviación estándar de los puntajes globales y por dominio, odds ratios ajustados e intervalos de confianza).

Riesgos de sesgo individuales de los estudios. El riesgo de sesgo de los estudios incluidos se evaluó utilizando la herramienta Effective Public Health Practice Project (EPHPP) para estudios cuantitativos que tiene seis componentes: sesgo de selección, diseño de estudio, factores confundentes, enmascaramiento, métodos de recopilación de datos y retiros / abandonos. Basándonos en la calificación de cada componente metodológico, el artículo era clasificado como fuerte, moderado o débil, obteniéndose una calificación global según el número de componentes determinados como débiles $(0,10>1)$.

Síntesis de los Datos. El resultado principal fue el impacto en la CVRSO medido a través de instrumentos validados. Cuando los estudios incluidos entregaban los resultados de CVRSO como puntajes (variables continuas), se calculó la diferencia de medias (MD) o la diferencia de medias estandarizadas (SMD) entre el grupo de individuos con caries y los controles y se agruparon en un metaanálisis. La SMD fue utilizada para combinar los datos de estudios que utilizan distintos instrumentos para evaluar CVRSO. Cuando los autores informaron los datos de CVRSO como una variable dicotómica que describe la presencia o ausencia de cualquier impacto negativo, se calcularon y agruparon los odds ratios (OR) ajustados. Se combinaron los OR ajustados, publicados en los estudios, utilizando el método de varianza genérica inversa.

Para todas las medidas, se realizaron diagramas de bosque que muestran el resumen y el IC del $95 \%$ estimado en los metaanálisis, junto con los resultados de los estudios individuales. Se utilizó un modelo de efectos aleatorios (método DerSimonian-Laird), debido a que esperábamos variaciones en los efectos producto de las diferencias en las poblaciones incluídas en los diferentes estudios, los cuestionarios y métodos empleados.
Cuando fue posible, realizamos un análisis de subgrupos de acuerdo al cuestionario utilizado para evaluar CVRSO, como posible fuente de heterogeneidad clínica. Además, planeamos realizar análisis de sensibilidad excluyendo los estudios que utilizaron cuestionarios diseñados para adultos y con calidad metodológica baja.

La heterogeneidad entre los estudios se evaluó usando el estadístico 12 categorizado de la siguiente manera: $<30 \%$ no es importante; $30 \%-50 \%$ moderado; $50 \%-75 \%$ sustancial; y $75 \%-100 \%$ considerable (Higgins \& Green, 2018) . Para explorar el posible sesgo de publicación, se planificó un gráfico en embudo cuando el número de estudios combinados fue $\geq 10$. El software utilizado fue Review Manager 5.3 (Cochrane IMS, Copenhague, Dinamarca).

\section{RESULTADOS}

Selección de estudios. La Figura 1 muestra un diagrama de flujo de los estudios. Tras la búsqueda sistemática fueron identificadas 1.152 citas. Se excluyeron 249 duplicados, quedando 903 artículos para la revisión de títulos, 120 para la revisión de los resúmenes, posteriormente 53 para la revisión del artículo completo, de los cuales 9 fueron excluidos porque incluían población preescolar, 3 no evaluaban CVRSO en caries, 3 no contaban con un grupo control, 3 no eran formatos originales y 1 empleaba un instrumento genérico para evaluar CVRSO. Finalmente 34 artículos fueron incluidos. El acuerdo entre los dos revisores durante el proceso de selección fue bueno ( $k=0,90$; IC95 \% 0,85-0,94).

Características de los estudios. Las principales características de los estudios incluídos se presentan en la Tabla I (en orden alfabético). Los 34 artículos correspondientes a 29 estudios incluyeron 18.554 participantes, todos publicados después de 2007. Veintiocho fueron estudios de corte transversal que comparaban grupos con y sin caries solo uno fue un estudio de cohorte. La mayoría de los estudios (13/29) se llevaron a cabo en Brasil, 3 en India, 2 en Perú, 2 en Nueva Zelanda, un estudio en Kuwait, Iran, Argentina, Australia, México, Nigeria, Tailandia, China y EEUU. El criterio de diagnóstico de caries de la OMS fue el más comúnmente aplicado (27 estudios) solo en dos se empleó el método ICDAS. La escala más utilizada para evaluar CVRSO fue la CPQ 11-14 en 18 estudios, seguida de la Child-OIDP en 6 , la OHIP con 4 y la OIDP con sólo un estudio. 


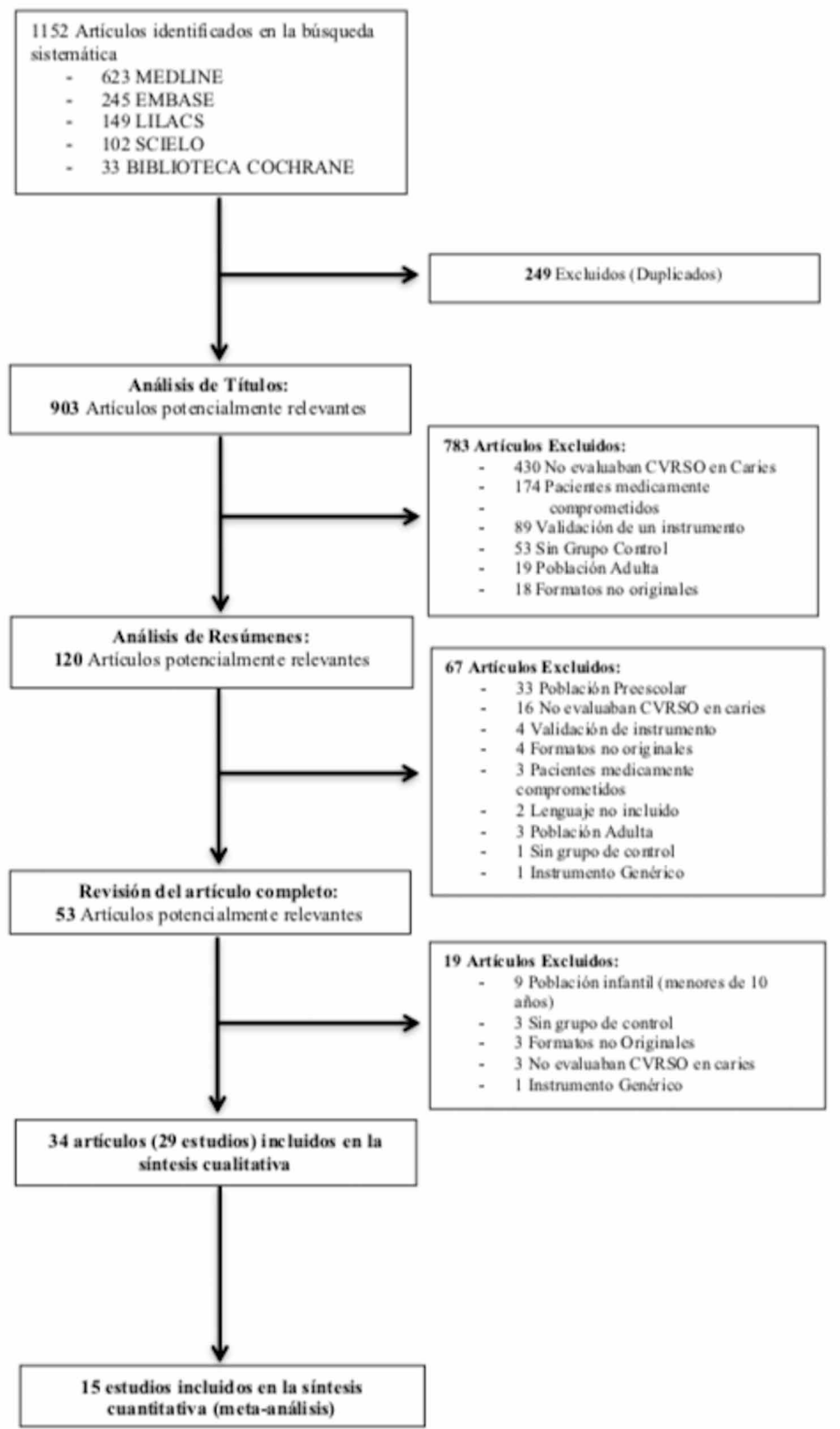

Fig. 1. Diagrama de flujo de la revisión sistemática de la literatura. do de recolección de datos" fueron los componentes mejores evaluados (mayoritariamente calificados como fuertes), debido a que las muestras fueron seleccionadas al azar principalmente de colegios y todos usaron instrumentos validados y confiables para evaluar CVRSO (uno de los requisitos de inclusión), considerada el resultado primario. El "Diseño del estudio" fue el parámetro peor evaluado siendo calificado mayoritariamente como débil, debido a que la mayoría de los estudios eran de corte transversal, excepto por uno de cohorte calificado como fuerte. El riesgo de sesgo de los "factores confundentes" fue calificado mayoritariamente como débil. Nueve estudios ajustaron los factores confundentes en su análisis para todas las variables clínicas y socioeconómicas importantes como traumatismos dentoalvoelares, maloclusiones, nivel socioeconómico y nivel educacional de los padres. El resto de los estudios fue clasificado como moderado o débil, debido a que menos del $80 \%$ de los factores de confusión importantes fueron controlados o descritos. Finalmente, el "enmascaramiento" fue calificado como moderado, debido a que la mayoría de los estudios no indicaban si el examen oral y la administración del cuestionario para evaluar CVRSO fue realizado por un investigador que no participó de los exámenes. Tampoco reportaron si los pacientes se encontraban enmascarados a la pregunta de investigación.

Impacto global en la CVRSO. Para variables continuas, ocho estudios (1.416 pacientes con caries y 1.432 pacientes sin caries) muestran que los pacientes con caries tienen una mayor probabilidad de presentar un impacto en la CVRSO, que aquellos libres de caries, sin diferencias significativas entre los grupos (SMD: 0,34; $95 \%$ IC: $-0,04-0,73 ;$ I2=95 \%) (Fig. 3).

Seis estudios fueron considerados en el metaanálisis construido con los datos ajustados del impacto de la caries sobre la CVRSO (Fig. 4) (OR: 2,50; IC 95 \%: 1,47-4,26; I2=93 \%). Como no todos los estudios incluidos utilizaban la misma escala para evaluar CVRSO, se crearon 3 subgrupos por cada instrumento empleado (OIDP, OHIP, CPQ11-14). 


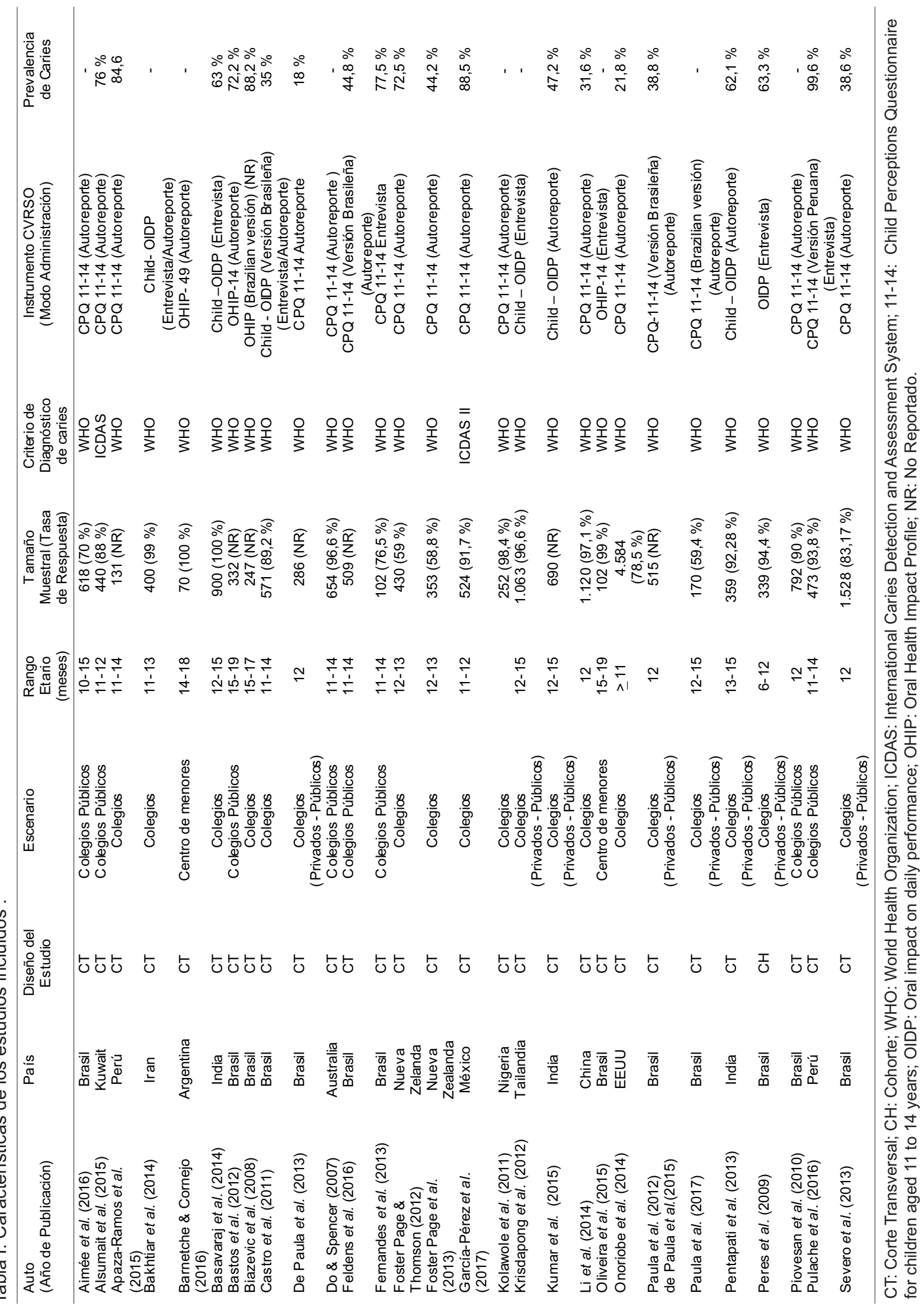




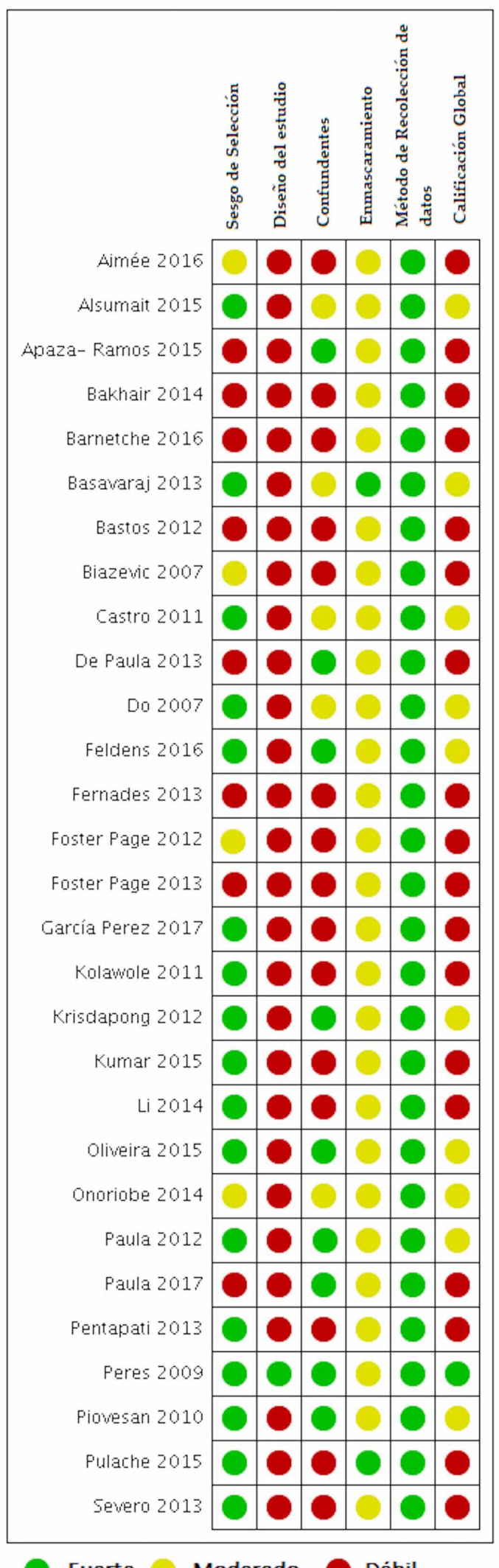

Fuerte

Moderado

Débil

Fig. 2. Riesgo de sesgo de los estudios incluídos.

Dominios Afectados. Siete estudios fueron incluídos en el metaanálisis de los principales dominios evaluados en las escalas de CVRSO (sintomático, funcional, sicológico y social) (Fig. 5). Todos los estudios considerados utilizaban la escala CPQ11-14 con 1.423 pacientes y 1.621 controles. Todos los dominios evaluados se ven afectados en los pacientes con caries, siendo los más comprometidos el funcional $(M D=0,74$; IC $95 \%: 0,27-1,20)$ y psicológico (MD=0,73; IC $95 \%: 0,21-1,26)$.

Análisis de Sensibilidad. Se efectuaron análisis de sensibilidad para evaluar la influencia del riesgo de sesgo de los estudios, así como también la de las escalas empleadas para medir CVRSO, en el resultado de los diversos metaanálisis. Para eso se excluyeron los estudios que habían sido calificados como débiles con la herramienta EPHPP y los que usaban las escalas OIDP y OHIP para medir CVRSO debido a que originalmente fueron diseñadas para adultos.

El análisis de sensibilidad fue realizado excluyendo las escalas diseñadas para adultos corrobora el impacto global de las caries en la CVRSO (OR: 3,41; IC 95 \%: 1,27-9,19; I2=96 $\%)$. Del mismo modo al excluir los estudios de baja calidad el impacto sobre la CVRSO sigue el doble en relación a aquellos que no presentan caries (OR: 2,00; IC: 1,28-3,11; I2: $89 \%$ ).

Por último, para el análisis de sensibilidad según el dominio de CVRSO afectado, se descartaron los estudios que habían sido clasificados cómo débiles con la herramienta EPHPP. Al analizar los resultados se mantiene la tendencia que señala que todos los dominios se ven afectados por las caries siendo los más perjudicados el psicológico $(\mathrm{MD}=0,90$; IC $95 \%: 0,42-1,38$ ) y el funcional (MD=0,64; IC $95 \%: 0,23-$ $1,06)$. En ambos casos la heterogeneidad bajo considerablemente de un $64 \%$ y $62 \%$ respectivamente, a $0 \%$.

\section{DISCUSIÓN}

La evidencia que vincula las caries con la CVRSO ha sido publicada principalmente entre los años 2007 - 2017. Esta revisión sistemática identificó 29 estudios que cumplían con los criterios de inclusión establecidos con un total de 18.554 participantes, de los cuales se reportaban 8.325 pacientes con caries y 10.039 controles. Al evaluar las diferencias en las medias de las puntuaciones entre los grupos, estas diferencias no fueron significativas. Sin embargo, al evaluar las proporciones de pacientes que informaron cualquier impacto en la CVRSO, la caries dental parece impactar negativamente en la calidad de vida de los adolescentes (OR: 2.50; IC $95 \%$ : 1,47-4,26; $12=93 \%$ ). Dado que no se ha determinado una diferencia mínima importante para considerar una alteración significativa para el paciente, el punto de corte seleccionado para dicotomizar la 




Fig. 3. Diferencia de medias estandarizada sobre la puntuación total de CVRSO.

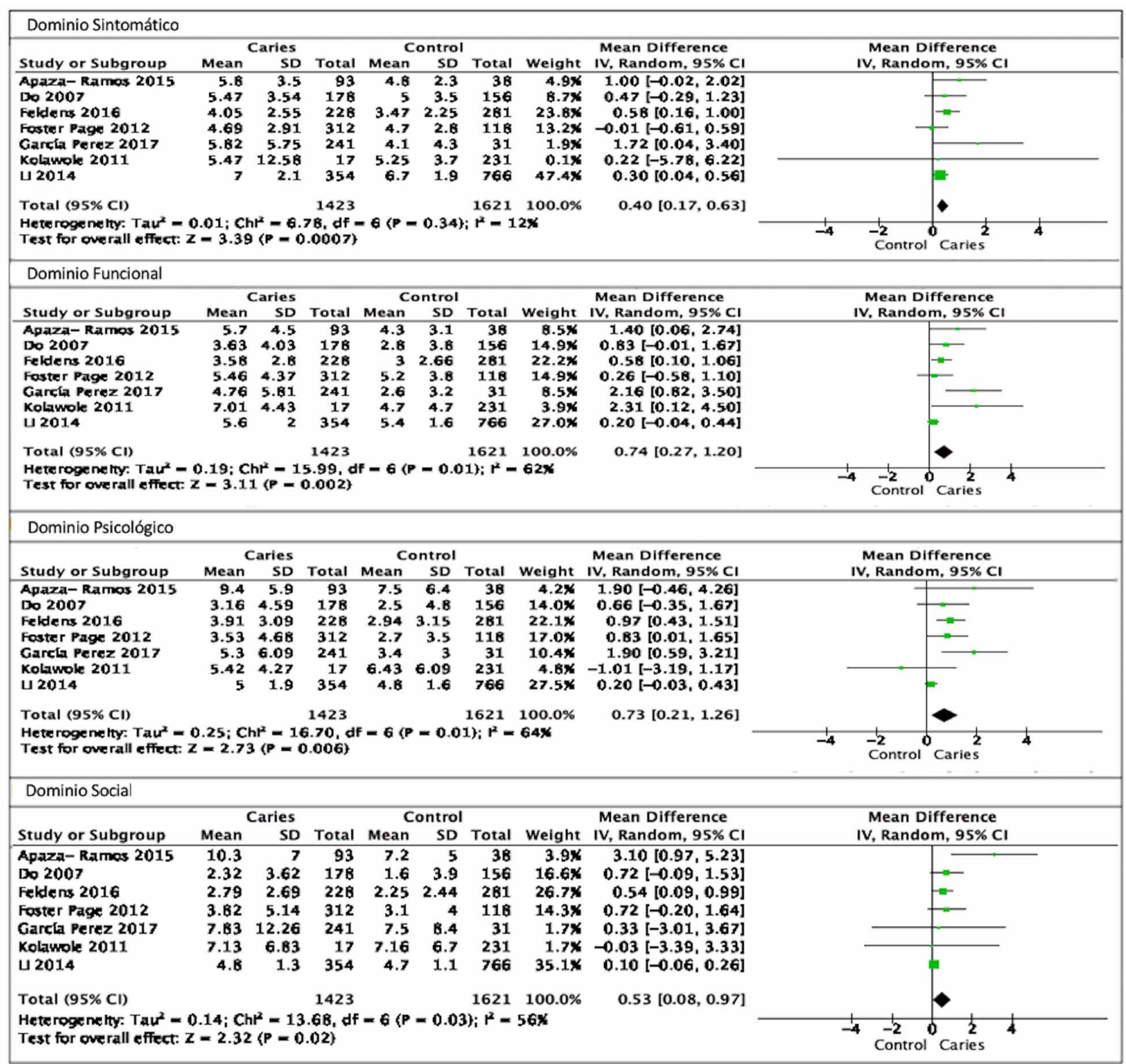

Fig. 4. Metaanálisis de los resultados globales ajustados de CVRSO. 


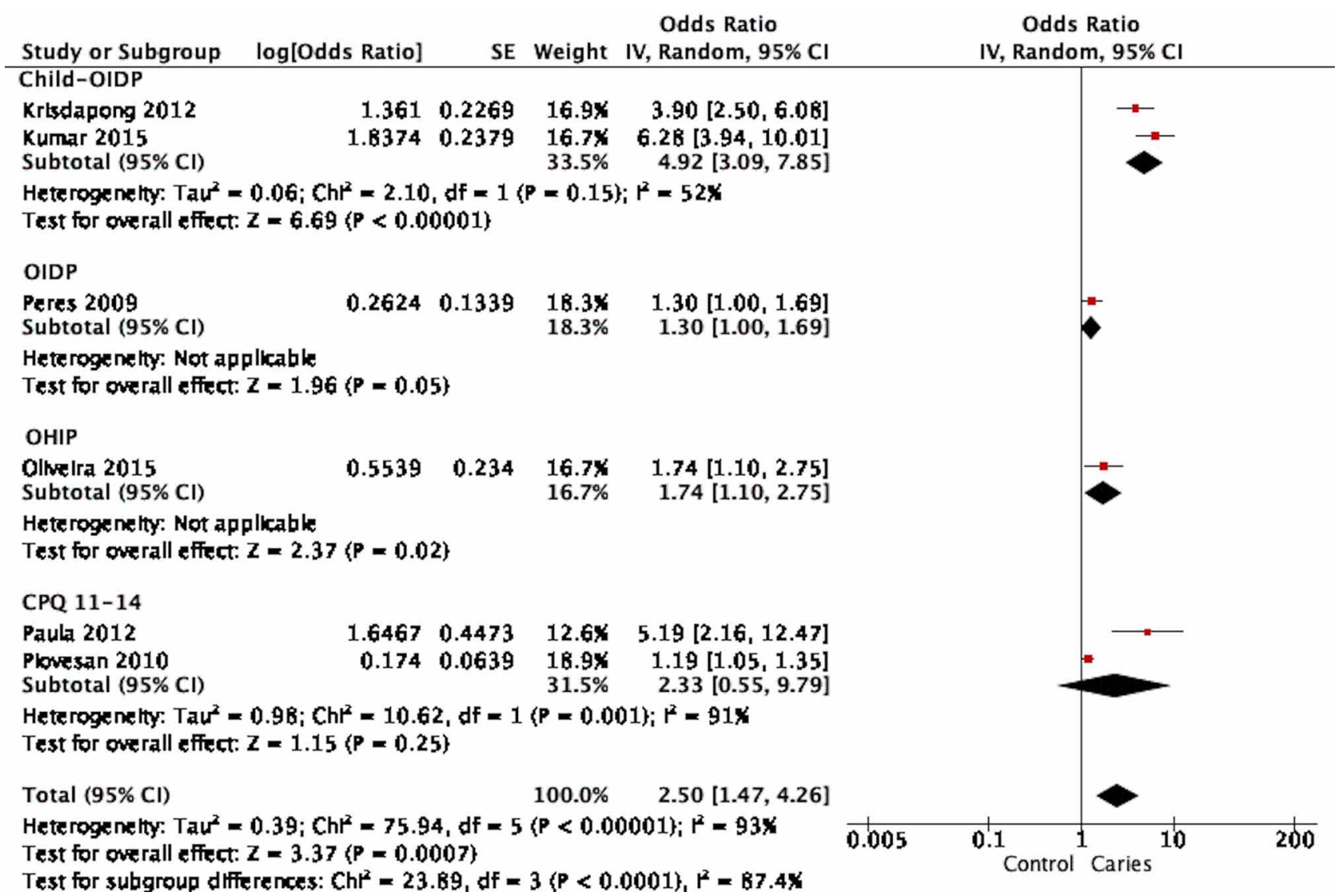

Fig. 5. Metaanálisis de las diferencias de medias de las puntuaciones de los dominios de CVRS.

variable continua podría afectar la prevalencia del impacto sobre CVRSO. Por lo tanto, la prevalencia podría estar sobreestimada en nuestra revisión, dado el punto de corte bajo usado por los estudios incluidos.

Por otro lado, al evaluar las proporciones, algunos de los estudios incluidos utilizaron instrumentos diseñados para población adulta para medir CVRSO en adolescentes. El análisis de sensibilidad realizado excluyendo los instrumentos diseñados para adultos, muestra un aumento del impacto en la calidad de CVRSO. Esto se puede deber a que los instrumentos diseñados para adultos son incapaces de medir adecuadamente la forma en que los niños experimentan la CVRSO subestimado el impacto, debido que su desarrollo cognitivo varía de tal manera que la redacción de los ítems, las dimensiones específicas, su relevancia y significado deben ser adaptados a su edad (McGrath et al., 2004)

Con respecto al modo de administración de las encuestas de CVRSO, la mayoría de los estudios utilizaron el autoreporte. La literatura señala que el mé- todo de administraciñon no afecta los resultado, independiente del método utilizado (entrevista personal, entrevista telefónica o cuestionario autoadministrado) (Mtaya et al., 2007; Castro et al., 2008; Misrachi et al., 2009).

Nuestros resultados también destacan que cada uno, de los cuatro dominios evaluados en las escalas utilizadas para medir calidad de vida relacionada con salud oral, se veían comprometidos, siendo los más afectados el dominio funcional y psicológico, lo que coincide con lo observado en otros estudios (Gherunpong et al., 2004; Tubert-Jeannin et al., 2005; Barbosa \& Gavião 2008). El análisis de sensibilidad confirmó estos resultados, disminuyendo la heterogeneidad a $0 \%$.

El dominio funcional se relaciona con problemas para hablar y masticar, mientras que el psicológico es consecuencia del dolor producido por las caries no tratadas. Esto genera malestar e irritación pudiendo alterar el sueño de los pacientes afectados (Yusuf et al., 2006). 
Es importante mencionar que identificamos algunas limitaciones en nuestro proceso de revisión. Primero, están aquellas derivadas de la naturaleza sistemática de la revisión, a pesar de considerar las bases de datos más importantes en el campo de las ciencias de la salud, es posible que no hayamos identificado todos los artículos para evaluar el impacto de la caries en la CVRSO de los adolescentes. Sin embargo, creemos que esto se minimizó gracias a la delicada estrategia de búsqueda utilizada, la búsqueda manual adicional de referencias, en conjunto a un doble proceso de revisión independiente realizado con posterioridad. Segundo, cuando hicimos la búsqueda, uno de los límites era el idioma, afortunadamente solo dos artículos fueron descartados bajo este criterio de exclusión, uno en alemán y otro en chino. En tercer lugar, es importante mencionar, que la validez interna del resumen proporcionado por un metaanálisis, depende de la calidad de los estudios primarios, la cual en general se calificó como débil. Por último, los resultados obtenidos deben considerarse con cautela, debido a la alta heterogeneidad obtenida, ya que podrían sobreestimarse los resultados.

A pesar de que el diseño experimental no es posible en este campo, se necesitan estudios prospectivos de cohorte para evaluar con la mejor evidencia científica el impacto de las caries en la CVRSO de los pacientes. Se debe tener precaución al generalizar los resultados, ya que la mayoría de los estudios provienen de poblaciones brasileñas con bajos niveles socioeconómicos. Finalmente, al no tener más de 10 estudios para agrupar en los diversos metaanálisis realizados, no se construyeron gráficos de embudo para explorar posibles sesgos de publicación.

Los hallazgos de esta revisión pueden contribuir para establecer las prioridades en la toma de decisiones clínicas con respecto al tratamiento dental de adolescentes. Además, estos resultados son relevantes porque ayudan a recalcar la importancia de un examen oral precoz, considerando la alta prevalencia de caries y su impacto en la calidad de vida. Teniendo esto en cuenta, tal vez se enfrente y planifique el tratamiento de esta enfermedad con un enfoque multidisciplinario, debido a que uno de los parámetros más afectados en la CVRSO de la población en estudio, aparte del aspecto funcional, fue el dominio psicológico.
MATAMALA-SANTANDER, A.; RIVERA-MENDOZA, F. \& ZAROR, C. Impact of caries on oral health related quality of life in adolescents: A systematic review and meta-analysis. Int. J. Odontostomat., 13(2):219-229, 2019.

ABSTRACT: Caries is an important public health problem, given its high prevalence and consequences in the quality of life of adolescents. The objective of this systematic review and meta-analysis is to evaluate the impact of dental caries on the Oral Health related Quality of Life(OHRQoL) in adolescents. A systematic search was carried out in the MEDLINE, EMBASE, Cochrane, ScieLo and Lilacs databases. Primary studies published in English, Spanish and Portuguese were included to evaluate OHRQoL in adolescents with caries using validated instruments. The selection process and data extraction were carried out by two researchers independently. The Effective Public Health Practice Project tool was used for the quality assessment. Random effect models were used to estimate the combined effect for continuous and categorical data. The combined search identified 1,152 articles, of which 29 studies (34 articles) met the inclusion criteria. Twenty-eight studies had a cross-sectional design and only one was cohort. The risk of bias was classified in most cases as weak (18/29). Patients with caries were significantly more likely to report any impact on OHRQoL than controls (OR $=2.50,95 \% \mathrm{Cl}: 1.47-4.26)$. The most affected domains were functional (Mean difference $(\mathrm{MD})=0.74,95 \% \mathrm{Cl}: 0.27-1.20)$ and psychological $(\mathrm{MD}=$ $0.73,95 \% \mathrm{Cl}: 0.21-1.26)$. This review provides evidence that caries has a negative impact on the OHRQoL in adolescents.

KEY WORDS: dental caries, quality of life, adolescents.

\section{REFERENCIAS BIBLIOGRÁFICAS}

Aimée, N. R.; van Wijk, A. J.; Maltz, M.; Varjão, M. M.; Mestrinho, H. D. \& Carvalho, J. C. Dental caries, fluorosis, oral health determinants, and quality of life in adolescents. Clin. Oral Investig., 21(5):1811-20, 2017.

Alsumait, A.; EISalhy, M.; Raine, K.; Cor, K.; Gokiert, R.; AlMutawa, S. \& Amin, M. Impact of dental health on children's oral health-related quality of life: a cross-sectional study. Health Qual. Life Outcomes, 13:98, 2015.

Apaza-Ramos, S.; Torres-Ramos, G.; Blanco-Victorio, D. J.; Antezana-Vargas, V. \& Montoya-Funegra, J. Influencia de los factores sociodemográficos, familiares y el estado de la salud bucal en la calidad de vida de adolescentes peruanos. Rev. Estomatol. Herediana, 25(2):87-99, 2015.

Baker, S. R. Testing a conceptual model of oral health: a structural equation modeling approach. J. Dent. Res., 86(8):708-12, 2007.

Bakhtiar, M.; Mohammadi, T. M.; Hajizamani, A. \& Vossoughi, M. Association of oral health indicators with quality-of-life related to oral health among Iranian adolescent. J. Int. Oral Health, 6(6):5-9, 2014.

Barbosa, T. S. \& Gavião, M. B. Oral health-related quality of life in 
children: part II. Effects of clinical oral health status. A systematic review. Int. J. Dent. Hyg., 6(2):100-7, 2008.

Barnetche, M. M. \& Cornejo, L. S. Experiencia de caries y calidad de vida de jóvenes en situación de encierro. Rev. Salud Publica, 18(5):816826, 2016.

Basavaraj, P.; Sunil, M. K.; Nagarajappa, R; Ashish, S. \& Ramesh, G. Correlation between oral health and Child-OIDP index in 12and 15-year-old children from Modinagar, India. Asia Pac. J. Public Health, 26(4):390-400, 2014.

Bastos, R. S.; Carvalho, E. S.; Xavier, A.; Caldana, M. L.; Bastos, J. R. \& Lauris, J. R. Dental caries related to quality of life in two Brazilian adolescent groups: a cross-sectional randomised study. Int. Dent. J., 62(3):137-43, 2012.

Biazevic, M. G.; Rissotto, R. R.; Michel-Crosato, E.; Mendes, L. A. \& Mendes, M. O. Relationship between oral health and its impact on quality of life among adolescents. Braz. Oral Res., 22(1):3642, 2008.

Casas López, M. J. Calidad de vida del adolescente. Rev. Cuba. Pediatr., 82(4):112-6, 2010.

Castro, R. A.; Cortes, M. I.; Leão, A. T.; Portela, M. C.; Souza, I. P.; Tsakos, G.; Marcenes, W. \& Sheiham, A. Child-OIDP index in Brazil: cross-cultural adaptation and validation. Health Qual. Life Outcomes, 6:68, 2008.

Castro, R. de A.; Portela, M. C.; Leão, A. T. \& de Vasconcellos, M. T. Oral health-related quality of life of 11- and 12-year-old public school children in Rio de Janeiro. Community Dent. Oral Epidemiol., 39(4):336-44, 2011.

Corrêa-Faria, P.; Paixão-Gonçalvez, S.; Paiva, S. M.; Martins-Júnior, P. A.; Vieira-Andrade, R. G.; Marques, L. S. \& Ramos-Jorge, M. L. Dental caries, but not malocclusion or developmental defects, negatively impacts preschoolers' quality of life. Int. J. Paediatr. Dent., 26(3):211-9, 2016.

de Paula, J. S.; Ambrosano, G. M. \& Mialhe, F. L. Oral disorders, socioenvironmental factors and subjective perception impact on children's school performance. Oral Health Prev. Dent., 13(3):219-26, 2015.

de Paula, J. S.; Leite, I. C.; de Almeida, A. B.; Ambrosano, G. M. \& Mialhe, F. L. The impact of socioenvironmental characteristics on domains of oral health-related quality of life in Brazilian schoolchildren. B. M. C. Oral Health, 13:10, 2013.

Do, L. G. \& Spencer, A. Oral health-related quality of life of children by dental caries and fluorosis experience. J. Public Health Dent., 67(3):132-9, 2007.

Feldens, C. A.; Ardenghi, T. M.; Dos Santos Dullius, A. I.; VargasFerreira, F.; Hernandez, P. A. \& Kramer, P. F. Clarifying the impact of untreated and treated dental caries on oral health-related quality of life among adolescents. Caries Res., 50(4):414-21, 2016.

Fernandes, M. L. M. F.; Moura, F.; Gamadiel, K. \& Correa-Faria, P. Dental caries and need of orthodontic treatment: impact on the quality of life of schoolchildren. Pesq. Bras. Odontopediatr. Clin. Integr., 13(1):37-43, 2013.

Foster Page, L. A. \& Thomson, W. M. Caries prevalence, severity, and 3-year increment, and their impact upon New Zealand adolescents' oral-health-related quality of life. J. Public Health Dent., 72(4):287-94, 2012.

Foster Page, L. A.; Thomson, W. M.; Jokovic, A. \& Locker, D. Epidemiological evaluation of short-form versions of the Child Perception Questionnaire. Eur. J. Oral Sci., 116(6):538-44, 2008.

Foster Page, L. A.; Thomson, W. M.; Jokovic, A. \& Locker, D. Validation of the Child Perceptions Questionnaire (CPQ 11-14). J. Dent. Res., 84(7):649-52, 2005.

Foster Page, L. A.; Thomson, W. M.; Ukra, A. \& Baker, S. R. Clinical status in adolescents: is its impact on oral health-related quality of life influenced by psychological characteristics? Eur. J. Oral Sci., 121(3 Pt. 1):182-7, 2013.

García-Pérez, Á.; Irigoyen-Camacho, M. E.; Borges-Yáñez, S. A.;
Zepeda-Zepeda, M. A.; Bolona-Gallardo, I. \& Maupomé, G. Impact of caries and dental fluorosis on oral health-related quality of life: a cross-sectional study in schoolchildren receiving water naturally fluoridated at above-optimal levels. Clin. Oral Investig., 21(9):2771-80, 2017.

Garcia, R.; Borrelli, B.; Dhar, V.; Douglass, J.; Gomez, F. R.; Hieftje K.; Horowitz, A.; Li, Y.; Ng, M. W.; Twetman, S.; et al. Progress in early childhood caries and opportunities in research, policy, and clinical management. Pediatr. Dent., 37(3):294-9, 2015.

Gherunpong, S.; Tsakos, G. \& Sheiham, A. Developing and evaluating an oral health-related quality of life index for children; the CHILD-OIDP. Community Dent. Health, 21(2):161-9, 2004.

Glick, M.; Monteiro da Silva, O.; Seeberger, G. K.; Xu, T.; Pucca, G.; Williams, D. M.; Kess, S.; Eiselé, J .L. \& Séverin, T. FDI Vision 2020: shaping the future of oral health. Int. Dent. J., 62(6):27891, 2012.

Higgins, J. P. T. \& Green, S. (Eds.). Cochrane Handbook for Systematic Reviews of Interventions. Version 5.1.0. Londres, The Cochrane Collaboration, 2018. Disponible en: http:// handbook.cochrane.org

Jokovic, A.; Locker, D.; Stephens, M.; Kenny, D.; Tompson, B. \& Guyatt, G. Validity and reliability of a questionnaire for measuring child oral-health-related quality of life. J. Dent. Res., 81(7):45963, 2002

Kolawole, K. A.; Otuyemi, O. D. \& Oluwadaisi, A. M. Assessment of oral health-related quality of life in Nigerian children using the Child Perceptions Questionnaire (CPQ 11-14). Eur. J. Paediatr. Dent., 12(1):55-9, 2011.

Krisdapong, S.; Prasertsom, P.; Rattanarangsima, K. \& Sheiham, A. Relationships between oral diseases and impacts on Thai schoolchildren's quality of life: evidence from a Thai national oral health survey of 12- and 15-year-olds. Community Dent. Oral Epidemiol., 40(6):550-9, 2012.

Krisdapong, S.; Prasertsom, P.; Rattanarangsima, K. \& Sheiham, A. School absence due to toothache associated with sociodemographic factors, dental caries status, and oral healthrelated quality of life in 12- and 15-year-old Thai children. J. Public Health Dent., 73(4):321-32, 2013.

Kumar, S.; Kumar, A.; Badiyani, B.; Kumar, A.; Basak, D. \& Ismail, M. B. Oral health impact, dental caries experience, and associated factors in 12-15-year-old school children in India. Int. J. Adolesc. Med. Health., 29(2), 2015.

Li, Y. J.; Gao, Y. H. \& Zhang, Y. The impact of oral health status on the oral health-related quality of life (OHRQoL) of 12-year-olds from children's and parents' perspectives. Community Dent. Health, 31(4):240-4, 2014.

Martins, M. T.; Sardenberg, F.; Vale, M. P.; Paiva, S. M. \& Pordeus, I. A. Dental caries and social factors: impact on quality of life in Brazilian children. Braz. Oral Res., 29(1):00310, 2015.

McGrath, C.; Broder, H. \& Wilson-Genderson, M. Assessing the impact of oral health on the life quality of children: implications for research and practice. Community Dent. Oral Epidemiol., 32(2):81-5, 2004.

Misrachi, C.; Ríos, M.; Morales, I.; Urzúa, J. P. \& Barahona, P. Calidad de vida y condición de salud oral en embarazadas Chilenas e inmigrantes Peruanas. Rev. Peru. Med. Exp. Salud Pública, 26(4):455-61, 2009.

Moher, D.; Liberati, A.; Tetzlaff, J.; Altman, D. G. \& PRISMA Group. Preferred reporting items for systematic reviews and metaanalyses: the PRISMA statement. PLoS Med., 6(7):e1000097, 2009.

Montero, J.; Costa, J.; Bica, I. \& Barrios, R. Caries and quality of life in portuguese adolescents: Impact of diet and behavioural risk factors. J. Clin. Exp. Dent., 10(3):e218-23, 2018.

Mtaya, M.; Åstrøm, A. N. \& Tsakos, G. Applicability of an abbreviated 
version of the Child-OIDP inventory among primary schoolchildren in Tanzania. Health Qual. Life Outcomes, 5:40, 2007.

Oliveira, D. C.; Ferreira, F. M.; Morosini, I. de A.; Torres-Pereira, C. C.; Martins Paiva, S. \& Fraiz, F. C. Impact of oral health status on the oral health-related quality of life of Brazilian male incarcerated adolescents. Oral Health Prev. Dent., 13(5):41725, 2015.

Onoriobe, U.; Rozier, R. G.; Cantrell, J. \& King, R. S. Effects of enamel fluorosis and dental caries on quality of life. J. Dent. Res., 93(10):972-9, 2014.

Palencia, L.; Espelt, A.; Cornejo-Ovalle, M. \& Borrell, C. Socioeconomic inequalities in the use of dental care services in Europe: what is the role of public coverage? Community Dent. Oral Epidemiol., 42(2):97-105, 2014.

Paula, J. S.; Cruz, J. N. D.; Ramires, T. G.; Ortega, E. M. M. \& Mialhe, F. L. Longitudinal impact of clinical and socioenvironmental variables on oral health-related quality of life in adolescents. Braz. Oral Res., 31:e70, 2017.

Paula, J. S.; Leite, I. C.; Almeida, A. B.; Ambrosano, G. M.; Pereira, A. C. \& Mialhe, F. L. The influence of oral health conditions, socioeconomic status and home environment factors on schoolchildren's self-perception of quality of life. Health Qual. Life Outcomes, 10:6, 2012.

Pentapati, K. C.; Acharya, S.; Bhat, M.: Krishna Rao, S. V. \& Singh, $S$. Oral health impact, dental caries, and oral health behaviors among the National Cadets Corps in South India. J. Investig. Clin. Dent., 4(1):39-43, 2013.

Peres, K. G.; Peres, M. A.; Araujo, C. L.; Menezes, A. M. \& Hallal, P. C. Social and dental status along the life course and oral health impacts in adolescents: a population-based birth cohort. Health Qual. Life Outcomes, 7:95, 2009.

Piovesan, C.; Antunes, J. L.; Guedes, R. S. \& Ardenghi, T. M. Impact of socioeconomic and clinical factors on child oral health-related quality of life (COHRQoL). Qual. Life Res., 19(9):1359-66, 2010.

Pulache, J.; Abanto, J.; Oliveira, L. B.; Bönecker, M. \& Porras, J. C. Exploring the association between oral health problems and oral health-related quality of life in Peruvian 11- to 14-year-old children. Int. J. Paediatr. Dent., 26(2):81-90, 2016.

Ramos-Jorge, J.; Alencar, B. M.; Pordeus, I. A.; Soares, M. E.; Marques, L. S.; Ramos-Jorge, M. L. \& Paiva, S. M. Impact of dental caries on quality of life among preschool children: emphasis on the type of tooth and stages of progression. Eur. J. Oral Sci., 123:88-95, 2015.

Severo Alves, L.; Dam-Teixeira, N., Susin, C. \& Maltz, M. Association among quality of life, dental caries treatment and intraoral distribution in 12-year-old South Brazilian schoolchildren. Community Dent. Oral Epidemiol., 41(1):22-9, 2013.

Sischo, L. \& Broder, H. L. Oral health-related quality of life: what, why, how, and future implications. J. Dent. Res., 90(11):126470, 2011.

Tubert-Jeannin, S.; Pegon-Machat, E.; Gremeau-Richard, C.; Lecuyer, M. M. \& Tsakos, G. Validation of a French version of the Child-OIDP index. Eur. J. Oral Sci., 113(5):355-62, 2005.

Turton, B. J.; Thomson, W. M.; Foster Page, L. A.; Saub, R. B. \& Razak, I. A. Validation of an oral health-related quality of life measure for Cambodian children. Asia Pac. J. Public Health, 27(2):NP2339-49, 2015.

Yusuf, H.; Gherunpong, S.; Sheiham, A. \& Tsakos, G. Validation of an English version of the Child-OIDP index, an oral health-related quality of life measure for children. Health Qual. Life Outcomes, 4:38, 2006.
Dirección para correspondencia:

Prof. Dr. Carlos Zaror Sánchez

Departamento de Odontopediatría y Ortodoncia

Facultad de Odontología

Universidad de La Frontera

Manuel Montt \#112, 4 piso

Temuco

CHILE

E-mail: carlos.zaror@ufrontera.cl

Recibido : 05-12-2018

Aceptado: 08-02-2019 